\title{
Corrigendum
}

\section{Corrigendum to "Null Geodesics and Strong Field Gravitational Lensing in a String Cloud Background"}

\author{
M. Sharif ${ }^{1}$ and Sehrish Iftikhar ${ }^{1,2}$ \\ ${ }^{1}$ Department of Mathematics, University of the Punjab, Quaid-e-Azam Campus, Lahore 54590, Pakistan \\ ${ }^{2}$ Department of Mathematics, Lahore College for Women University, Lahore 54000, Pakistan \\ Correspondence should be addressed to M. Sharif; msharif.math@pu.edu.pk
}

Received 2 July 2015; Accepted 16 July 2015

Copyright ( 2015 M. Sharif and S. Iftikhar. This is an open access article distributed under the Creative Commons Attribution License, which permits unrestricted use, distribution, and reproduction in any medium, provided the original work is properly cited. The publication of this article was funded by SCOAP ${ }^{3}$.

In the paper titled "Null Geodesics and Strong Field Gravitational Lensing in a String Cloud Background," recently, we have studied null geodesics and strong field gravitational lensing of a black hole with string cloud background [1]. References of some equations were missing in the paper titled "Null Geodesics and Strong Field Gravitational Lensing in a String Cloud Background." Here we add these references. Equations (10), (13), and (22) were originally derived in the following references:

Claudel, C. M., Virbhadra, K. S. and Ellis, G. F. R.: J. Math. Phys. 42(2001)818.

Virbhadra, K. S., Narasimha, D. and Chitre, S. M.: Astron. Astrophys. 337(1998)1.

Virbhadra, K. S. and Ellis, G. F. R.: Phys. Rev. D 65(2002)103004.

\section{References}

[1] M. Sharif and S. Iftikhar, "Null geodesics and strong field gravitational lensing in a string cloud background," Advances in High Energy Physics, vol. 2015, Article ID 635625, 9 pages, 2015. 

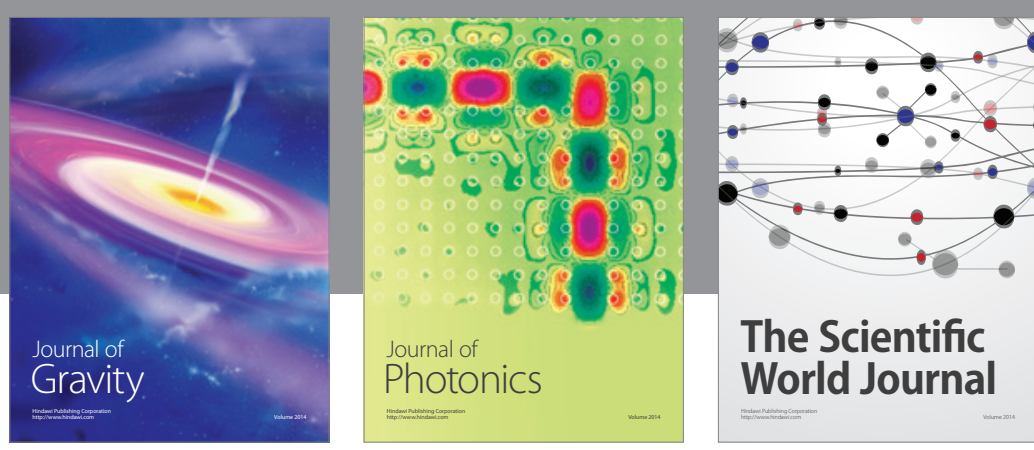

The Scientific World Journal
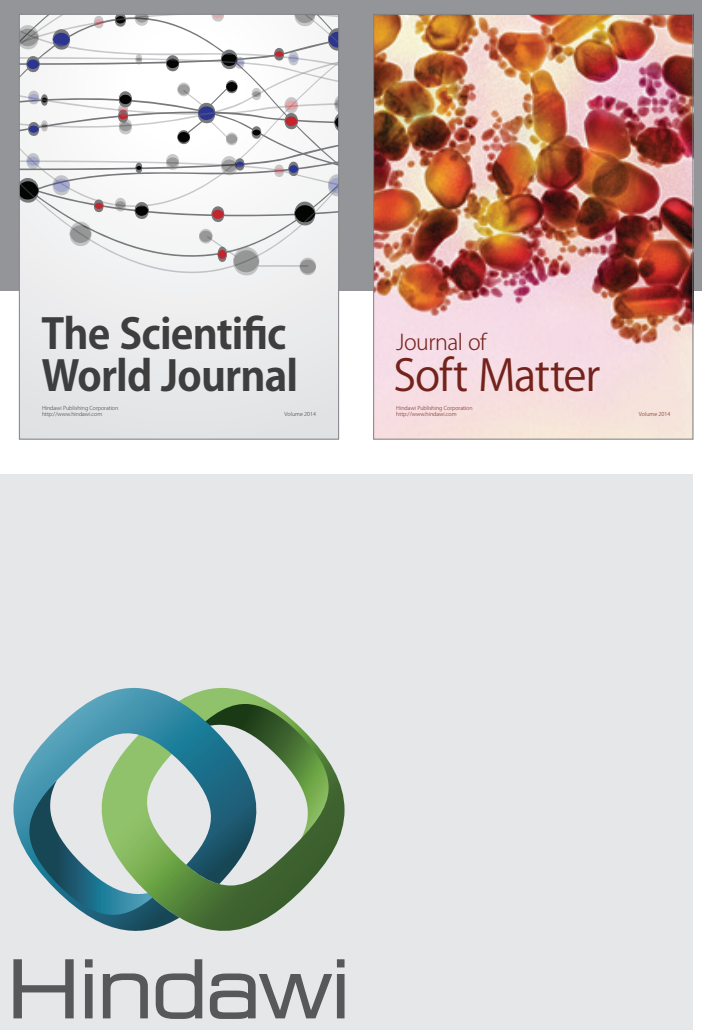

Submit your manuscripts at

http://www.hindawi.com

nternational Journal of

Statistical Mechanics
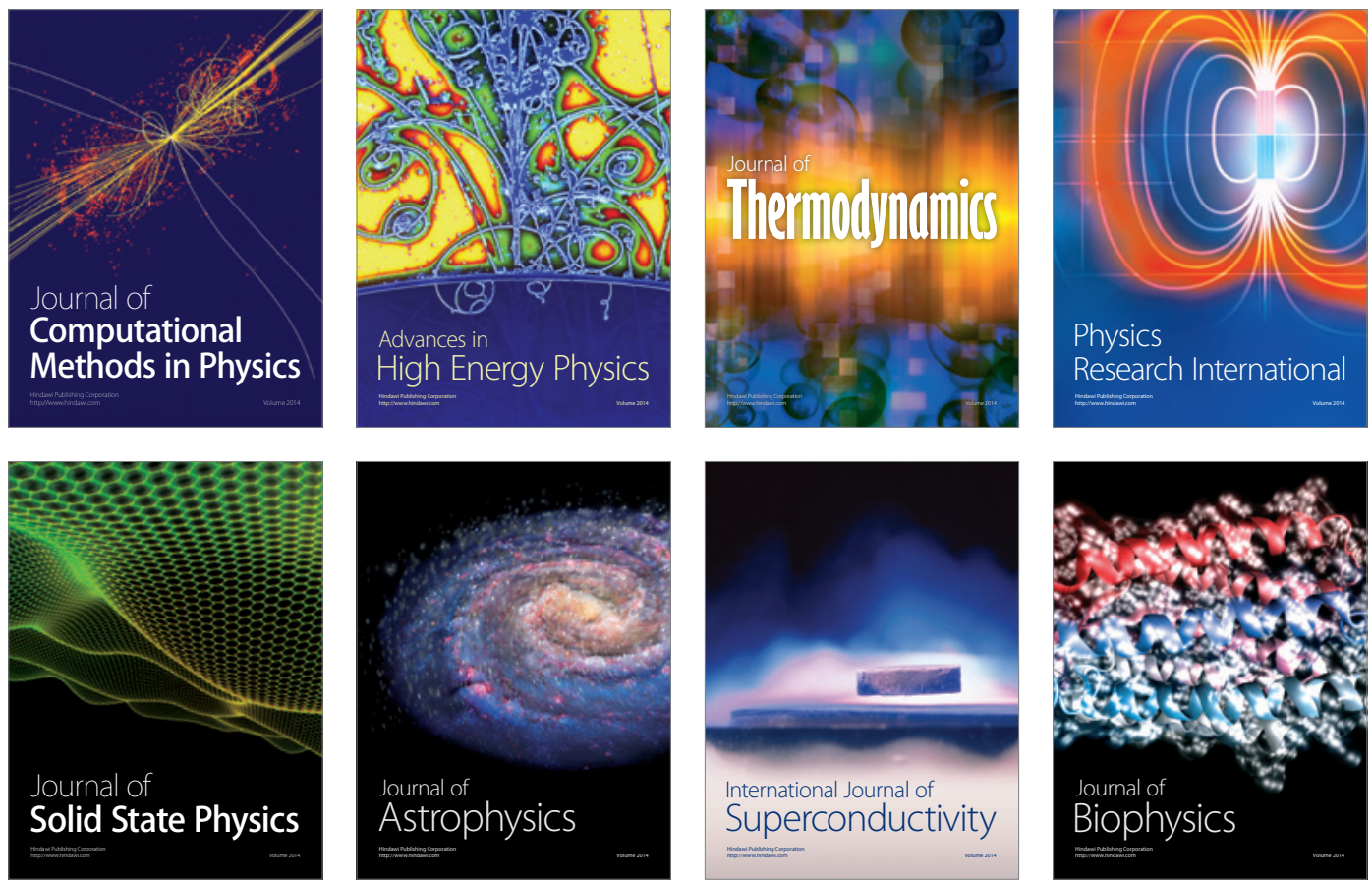
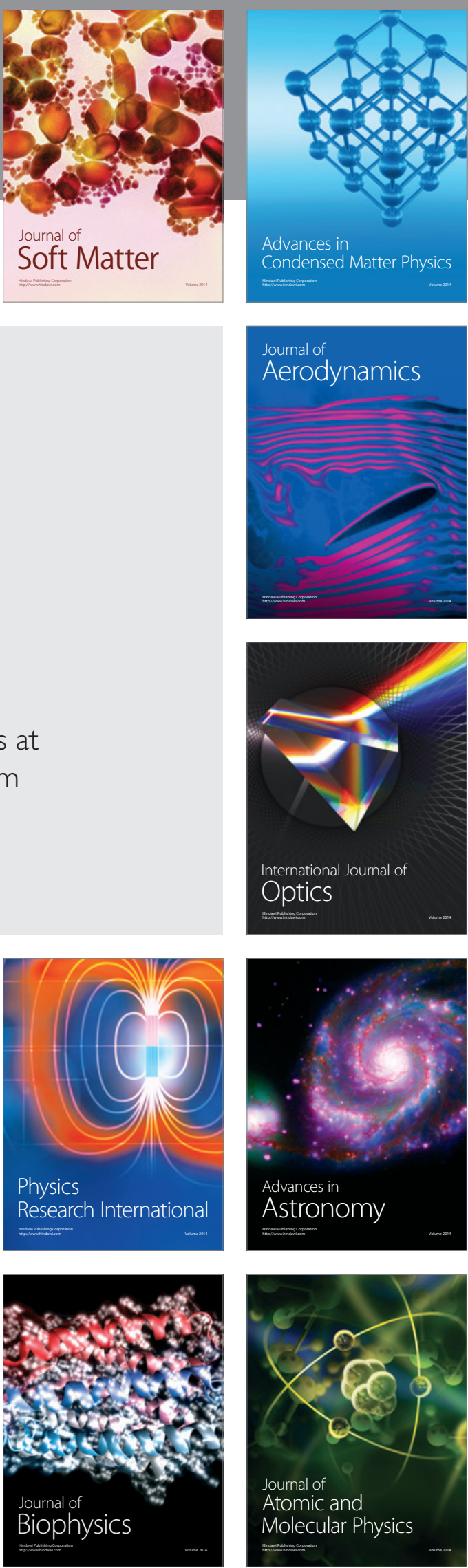